\title{
Factors related to hospital readmissions in people with spinal cord injury in South Africa
}

\author{
M K Mashola, ${ }^{1}$ MPhysT; S A S Olorunju, ${ }^{2} \mathrm{PhD} ; \mathrm{J}$ Mothabeng, ${ }^{1} \mathrm{PhD}$ \\ ${ }^{1}$ Department of Physiotherapy, School of Health Care Sciences, Faculty of Health Sciences, University of Pretoria, South Africa \\ ${ }^{2}$ Biostatistics Unit, South African Medical Research Council, Pretoria, South Africa
}

Corresponding author: M K Mashola (kholofelo.mashola@up.ac.za)

Background. People with spinal cord injury (PWSCI) face various challenges after being discharged from rehabilitation that can result in readmission to hospital. Little is known about readmission of PWSCI in South Africa (SA). Readmission is costly, interrupts community involvement and negatively affects quality of life.

Objectives. To investigate readmission rates within 5 years of rehabilitation, causes of readmission and factors related to readmission in PWSCI in Pretoria, SA.

Methods. We quantitatively analysed retrospective data gathered from files of patients admitted to a private rehabilitation facility in Pretoria between January 2008 and December 2012. Data were analysed using Stata 13 statistical software. Descriptive statistics were initially presented. Univariate logistic regression was used to identify individual factors that had significant association with the outcome measure (readmission). Thereafter, multivariate logistic regression was used to identify risk factors for readmission. The level of statistical significance was set at $p<0.5$.

Results. Data from 543 patient files were analysed. In total, 100 patients (18\%) were readmitted between January 2008 and December 2012. Twenty-eight of the 100 readmitted patients had a subsequent second readmission, 10 patients had a third readmission, and 2 patients were readmitted for a fourth time. The most common reason for readmission was secondary health conditions (SHCs) (80\%), followed by further rehabilitation, including gait rehabilitation (12\%). Eight patients (8\%) had undocumented reasons for readmission. The common SHCs in the first readmission were pressure ulcers (39\%), followed by urinary tract infections (12\%), deteriorating neurological status $(6 \%)$ and constipation (3\%). Patients with paraplegia had 2.3 times greater odds of readmission compared with tetraplegics $(p=0.000,95 \%$ CI 1.47 . 3.55). Those in the category T1 - T6 level of injury had 2.6 times greater odds of readmission ( $p=0.04,95 \%$ CI $1.04-6.71$, while those with incomplete spinal cord injury had 2.5 times greater odds ( $p=0.001,95 \%$ CI $1.44-4.46$ ).

Conclusions. Factors related to patient injury profile such as type, completeness and level of injury were associated with a significant risk of readmission. SHCs were the main cause of readmission, and there is a need for effective programmes for their prevention.

S Afr Med J 2019;109(2):107-111. DOI:10.7196/SAMJ.2019.v109i2.13344

People with spinal cord injury (PWSCI) spend extended periods of time in rehabilitation, where they learn to adjust to a new physical reality. ${ }^{[1]}$ After discharge from rehabilitation, some PWSCI are readmitted for various reasons. Readmission can be disruptive and undermine rehabilitation achievements, ultimately diminishing an individual's ability to live an active and independent life. ${ }^{[2]}$ Readmission rates of PWSCI can outpace the general population by as much as 2.6 times and are highest in the first year after discharge from rehabilitation. ${ }^{[3]}$ Within the first year after spinal cord injury (SCI), as many as $26-39 \%$ of PWSCI are readmitted, with rates ranging from 0.55 to 1.85 readmissions per year, declining to rates between 0.26 to 0.55 in subsequent years. ${ }^{[4]}$ Readmission rates may vary or decrease over time, depending on the cause of readmission. There are numerous studies on readmission rates after SCI, although there is little literature on readmissions in South Africa (SA); however, only a few have examined factors associated with readmission.

PWSCI are usually readmitted for secondary health conditions $(\mathrm{SHCs}){ }^{[2]}$ defined ${ }^{\left[{ }^{[3]}\right.}$ 'physical or psychological health conditions that are influenced directly or indirectly by the presence of a disability or underlying physical impairment?. PWSCI are prone to a variety of SHCs that impact negatively on health, emotional wellbeing, community participation and quality of life..$^{[3]}$ While readmission rates may vary across studies, the SHCs responsible for readmission are nearly always the same. ${ }^{[2]}$ The most common SHCs responsible for readmission in PWSCI are skin, genitourinary and respiratory complications. ${ }^{[3]}$ While readmissions due to skin and respiratory complications may vary over time, readmission rates for genitourinary SHCs tend to increase as the years living with SCI increase. ${ }^{[1]}$ Secondary health conditions do not exist in isolation but have the potential to exacerbate each other, creating a synergistic effect that can lead to serious overall health complications or even death. ${ }^{[3]}$ Although some readmissions may improve functional status and many readmissions are unavoidable, it is important to reduce the health burden to PWSCI by preventing readmissions. ${ }^{[1]}$

Over and above their health implications and impact on quality of life, SHCs pose a financial burden to PWSCI, contributing directly to $32 \%$ of medical costs in the first 2 years after injury. Pressure ulcers (PUs) are especially difficult to treat, leading to disproportionately long stays in hospital with high treatment costs. ${ }^{[3]}$

\section{Objectives}

There has been little research on reasons for hospital readmission after discharge from rehabilitation for PWSCI living in SA. We therefore investigated readmission rates of PWSCI after discharge from rehabilitation, reasons for readmission and factors related to readmission within 5 years after discharge from a private rehabilitation facility. 


\section{Methods \\ Design and materials}

We followed a quantitative design using a retrospective review to investigate readmission of PWSCI who were discharged from a private rehabilitation facility in the City of Tshwane metropolitan area centred on Pretoria, SA. We employed a non-probability consecutive sampling method, and all patient medical records that met the criteria (a primary or secondary diagnosis of paraplegia or tetraplegia and that contained demographic, injury profile, admission and discharge date information) were included in the study.

\section{Setting}

We identified potential patient files from the electronic database of a private rehabilitation facility in Pretoria. This multidisciplinary facility is one of three private facilities in Gauteng Province that specialise in SCI and adult neurology and manage SHCs after SCI.

\section{Procedure and data analysis}

After selecting patient files that met the aforementioned inclusion criteria, we sampled files of patients readmitted between January 2008 and December 2012. Patients who were readmitted were identified by recurring patient numbers. The recurring patient numbers were cross-checked with demographic details to ensure that they referred to the same readmitted patient. Data collected from the electronic database were saved on an Excel spreadsheet, version 2010 (Microsoft, USA), and coded for analysis. Age, gender, length of stay (LOS), type of injury, level and completeness of injury, number of readmissions, causes of readmissions and discharge status were coded and analysed descriptively using the statistical package Stata 13 (StataCorp, USA). Factors related to readmission were analysed using multivariable logistic regression with $95 \%$ confidence intervals (CIs). The level of statistical significance was set at $p<0.5$.

\section{Ethical considerations}

Permission to access patient files was formally granted by the private rehabilitation facility. Institutional ethical clearance was obtained from the Faculty of Health Sciences Research Ethics Committee, University of Pretoria (ref. no. 21/2014). Data collected were submitted to the Physiotherapy Department of the University of Pretoria and will be stored there until 2029.

\section{Results \\ Descriptive data}

Of a total of 1114 files retrieved, 543 met the study criteria. The ages of these patients ranged from 7 years to 87 years; $23 \%$ were 30 - 39 years old and the smallest group (3\%) comprised patients aged $<18$ years. Most of the patients were male (75\%, female $25 \%)$. Traumatic causes such as road accidents, violence, agricultural accidents and falls accounted for $50 \%$ of SCIs (non-traumatic causes $25 \%$, unknown cause $25 \%$ ). Most patients had paraplegia (61\%) and complete injuries (58\%). The most commonly documented neurological level of injury (NLI) was C5 - C8 (19\%). Thirty-three per cent of patients did not have NLI documented. Twenty-six per cent of the patients were able to use their wheelchairs independently on discharge, while $6 \%$ died in hospital (Table 1).

\section{Readmission data}

Of the 543 files investigated, $100(18 \%)$ indicated that the patient was readmitted between January 2008 and December 2012 (Table 2). Twenty-eight patients (28\%) had a subsequent second readmission and 10 patients a third readmission, and 2 patients were readmitted

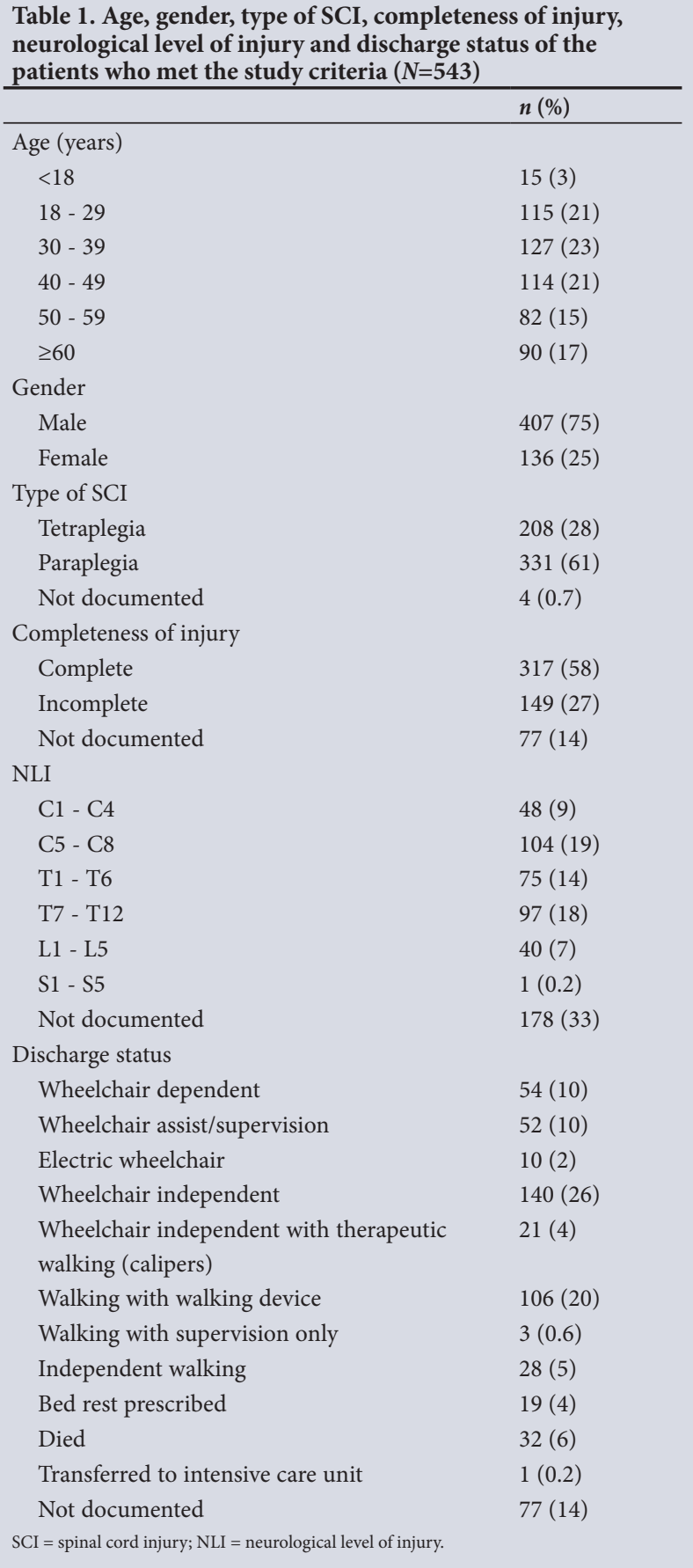

for a fourth time. The most common reason for readmission was SHCs (80\%), followed by further rehabilitation (including gait rehabilitation) (12\%). Eight patients (8\%) had undocumented reasons for readmission. The common SHCs in the first readmission were PUs (39\%), followed by urinary tract infections (UTIs) (12\%), deteriorating neurological status (6\%), constipation (3\%) and hip dislocations (2\%). Respiratory conditions were found to be the reason for readmission in only 4 single separate readmissions (Table 3 ).

PUs were also the most common SHCs in the second (39\%) and third (60\%) readmissions. UTIs were reported in $25 \%$ of second readmissions and $40 \%$ of third readmissions. PUs and hypertension each caused $50 \%$ of fourth readmissions (Table 3). In the first readmission, PUs affected more patients aged 18 - 39 years with C5 - 


\begin{tabular}{|c|c|}
\hline & $n(\%)$ \\
\hline \multicolumn{2}{|l|}{ Age (years) } \\
\hline$<18$ & $2(13)$ \\
\hline $18-29$ & $29(25)$ \\
\hline $30-39$ & $25(12)$ \\
\hline $40-49$ & $23(20)$ \\
\hline $50-59$ & $13(16)$ \\
\hline$\geq 60$ & $8(9)$ \\
\hline \multicolumn{2}{|l|}{ Gender } \\
\hline Male & $78(19)$ \\
\hline Female & $22(16)$ \\
\hline \multicolumn{2}{|l|}{ Type of SCI } \\
\hline Tetraplegia & $55(26)$ \\
\hline Paraplegia & $45(14)$ \\
\hline \multicolumn{2}{|l|}{ Completeness of injury } \\
\hline Complete & $78(25)$ \\
\hline Incomplete & $17(11)$ \\
\hline Not documented & $5(7)$ \\
\hline \multicolumn{2}{|l|}{ NLI } \\
\hline $\mathrm{C} 1-\mathrm{C} 4$ & $13(27)$ \\
\hline $\mathrm{C} 5-\mathrm{C} 8$ & $33(32)$ \\
\hline $\mathrm{T} 1$ - T6 & $11(15)$ \\
\hline T7 - T12 & $18(19)$ \\
\hline L1 - L5 & $5(13)$ \\
\hline S1 - S5 & 0 \\
\hline Not documented & $20(11)$ \\
\hline \multicolumn{2}{|l|}{ Discharge status } \\
\hline Wheelchair dependent & $17(35)$ \\
\hline Wheelchair assist/supervision & $16(31)$ \\
\hline Electric wheelchair & $4(40)$ \\
\hline Wheelchair independent & $32(23)$ \\
\hline $\begin{array}{l}\text { Wheelchair independent with therapeutic } \\
\text { walking (calipers) }\end{array}$ & $8(38)$ \\
\hline Walking with walking device & $5(5)$ \\
\hline Walking with supervision only & $0(0)$ \\
\hline Independent walking & $4(14)$ \\
\hline Bed rest prescribed & $2(11)$ \\
\hline Died & $2(6)$ \\
\hline Not documented & $10(13)$ \\
\hline $\mathrm{SCI}=$ spinal cord injury; $\mathrm{NLI}=$ neurol & \\
\hline
\end{tabular}

C8 complete tetraplegia than other groups. Patients with complete injuries (75\%) and tetraplegia (67\%) were most commonly admitted for UTI treatment. Deteriorating neurological status was most prevalent in patients with incomplete injuries (80\%) and tetraplegia (60\%). Respiratory complications (4\%) were only observed in patients with complete injuries and those with NLI above T6, and patients aged 40 - 49 years $(50 \%)$.

Of all the SHCs, PUs resulted in the longest LOS in the first (2 173 days), second (562 days) and third (585 days) readmissions. The longest LOS in the fourth readmission was associated with hypertension (91 days), followed by PUs (79 days). UTIs resulted in LOS of 78 and 40 days in the second and third readmissions, respectively. Twelve patients (12\%) were readmitted for further rehabilitation ( $n=2$, LOS 79 days) and gait rehabilitation $(n=10$, LOS 190 days). The latter readmissions were evenly distributed between tetraplegics and paraplegics ( $n=5$ each). Only 1 patient had a subsequent second readmission for further rehabilitation (LOS 11 days). Readmission for gait rehabilitation was more common in patients with complete injuries $(60 \%)$ than in patients with incomplete injuries (40\%). Reasons for readmission of 8 patients were not documented, resulting in 335 unaccounted hospital days (Table 3).

Readmission was found to be associated with completeness of injury, NLI and type of SCI at a 95\% CI. Patients with an incomplete SCI had 2.5 greater odds of readmission compared with those with complete SCI ( $p=0.001,95 \%$ CI $1.44-4.46)$, patients with NLI at T1 - T6 had 2.6 greater odds of readmission than others $(p=0.041$, $95 \%$ CI $1.04-6.71$ ), and patients with paraplegia had 2.3 greater odds of readmission than those with tetraplegia $(p=0.000,95 \%$ CI 1.47 - 3.55).

\section{Discussion}

This study found that $18.4 \%$ of patients were readmitted within the first 5 years after discharge from a private rehabilitation facility following an SCI. Patients had an average LOS of 34.8 days across all first readmission episodes. In contrast, a large multicentre study conducted in the USA found that $36.2 \%$ of patients were readmitted 1 year after SCI, with an average LOS of 15.5 days across all readmission episodes. ${ }^{[2]}$ De Jong et al ${ }^{[2]}$ documented readmission rates after rehabilitation in non-profit rehabilitation facilities, while we assessed readmission after rehabilitation in a private facility. In their study, ${ }^{[2]}$ patients who were discharged with low functional independence, assessed using the functional independence measure, had greater likelihood of readmission. Similarly, we found that patients who were wheelchair dependent (including those using electric wheelchairs) were readmitted more than patients who were wheelchair independent $(32 \%$ v. $24 \%)$. Patients who were able to walk at discharge were not readmitted with SHCs in our study. Most PWSCI who pursue active leisure activities do so by visiting friends and family, which may mean that they are wheelchair bound for relatively long periods of time. Extended wheelchair use is implicated in the development of PUs, especially if PWSCI do not follow pressure-relief practices. ${ }^{[7]}$

We noted lower overall readmission rates (18\%) than in international studies, ${ }^{[6]}$ but a high proportion $(28 \%)$ of those were readmitted at least twice for an average LOS of 28.8 days, compared with $12.5 \%$ in a US study. ${ }^{[2]}$ This may be explained by the different time frames of the two studies, as our study considered readmission over a period of 60 months, with initial discharge within 5 years, while De Jong et al. ${ }^{[2]}$ considered rehospitalisation within 12 months of rehabilitation discharge. Within our 60-month window, $10 \%$ of patients were readmitted three times (average LOS 62.5 days) and $2 \%$ were readmitted four times (average LOS 85 days). The high rate of subsequent readmissions and increasing days spent in hospital suggest that medical care, health promotion and SHC prevention in developing countries may be greatly outpaced by practices in developed countries. While subsequent readmission rates reduce over time after SCI, as supported by Cardenas et al., ${ }^{[6]}$ LOS steadily increases with each readmission. This may suggest that the pace of healing is slowed down with increasing development of SHCs. In our study, treatment for PUs caused disproportionately long hospital stays, accounting for a total of 2 173, 562, 585 and 79 days for the first, second, third and fourth readmissions, respectively. The average LOS for the management of PUs increased from 55.7 days in the first readmission to 79 days in the fourth. Management of PUs is complicated and the associated long hospital stays contribute to the financial burden of PWSCI. Despite protocols to reduce 


\begin{tabular}{|c|c|c|c|c|}
\hline Reason & $\begin{array}{l}\text { First }(N=100), \\
n(\mathrm{LOS})\end{array}$ & $\begin{array}{l}\text { Second }(N=28), \\
n(\text { LOS })\end{array}$ & $\begin{array}{l}\text { Third }(N=10), \\
n(\mathrm{LOS})\end{array}$ & $\begin{array}{l}\text { Fourth }(N=2), \\
n(\mathrm{LOS})\end{array}$ \\
\hline \multicolumn{5}{|l|}{ Integumentary (skin) } \\
\hline $\mathrm{PU}$ & $39(2$ 173) & $11(562)$ & $6(585)$ & $1(79)$ \\
\hline Burns & $1(37)$ & $1(45)$ & 0 & 0 \\
\hline \multicolumn{5}{|l|}{ Urinary system } \\
\hline UTI & $12(69)$ & $7(78)$ & $4(40)$ & 0 \\
\hline \multicolumn{5}{|l|}{ Central nervous system } \\
\hline Deteriorating neurological status & $6(167)$ & 0 & 0 & 0 \\
\hline Autonomic dysreflexia & $1(5)$ & 0 & 0 & 0 \\
\hline Severe pain & $1(49)$ & 0 & 0 & 0 \\
\hline Syringomyelia & $1(8)$ & 0 & 0 & 0 \\
\hline Vasovagal headaches & $1(10)$ & 0 & 0 & 0 \\
\hline Dementia & 0 & $1(16)$ & 0 & 0 \\
\hline \multicolumn{5}{|l|}{ Digestive system } \\
\hline Constipation & $3(51)$ & $2(13)$ & 0 & 0 \\
\hline Rectal bleeding & $1(7)$ & 0 & 0 & 0 \\
\hline Constipation and UTI & $1(9)$ & 0 & 0 & 0 \\
\hline Constipation and $\mathrm{PU}$ & $1(68)$ & 0 & 0 & 0 \\
\hline \multicolumn{5}{|l|}{ Respiratory system } \\
\hline Acute pulmonary insufficiency & $1(17)$ & 0 & 0 & 0 \\
\hline Bronchopneumonia & $1(8)$ & 0 & 0 & 0 \\
\hline Chest pains & $1(6)$ & 0 & 0 & 0 \\
\hline Pulmonary TB & $1(22)$ & 0 & 0 & 0 \\
\hline \multicolumn{5}{|l|}{ Muscular system } \\
\hline Bicipital tendinitis & $1(79)$ & 0 & 0 & 0 \\
\hline Low back pain & $1(5)$ & 0 & 0 & 0 \\
\hline Rotator cuff injury & $1(3)$ & 0 & 0 & 0 \\
\hline \multicolumn{5}{|l|}{ Skeletal system } \\
\hline Femur fracture & $1(10)$ & 0 & 0 & 0 \\
\hline Hip dislocation & $2(20)$ & 0 & 0 & 0 \\
\hline \multicolumn{5}{|l|}{ Surgical } \\
\hline Unspecified surgical procedure & $1(13)$ & 0 & 0 & 0 \\
\hline \multicolumn{5}{|l|}{ Cardiovascular system } \\
\hline Vitamin B deficiency & $1(44)$ & 0 & 0 & 0 \\
\hline Cellulitis & 0 & $2(30)$ & 0 & 0 \\
\hline Hypertension & 0 & 0 & 0 & $1(91)$ \\
\hline \multicolumn{5}{|l|}{ Reproductive system } \\
\hline Orchitis and epididymitis & 0 & $1(7)$ & 0 & 0 \\
\hline Testicular TB & 0 & $1(39)$ & 0 & 0 \\
\hline Vesicovaginal fistula & 0 & $1(5)$ & 0 & 0 \\
\hline \multicolumn{5}{|l|}{ Other causes of readmission } \\
\hline Further rehabilitation & $2(79)$ & $1(11)$ & 0 & 0 \\
\hline Gait rehabilitation & $10(190)$ & 0 & 0 & 0 \\
\hline Not documented & $8(335)$ & 0 & 0 & 0 \\
\hline
\end{tabular}

the development of PUs, they remain the most common SHCs contributing to readmissions, in our study and others. ${ }^{[1,2,6]}$ In the present study, 39\% of first readmissions were caused by PUs, similar to the finding of Joseph and Nilsson Wikmar ${ }^{[8]}$ that PUs caused 30\% of SHCs in the Western Cape Province, SA. The most common SHCs may vary from one study to another, as SHCs are dependent on demographic and injury-related factors. ${ }^{[9]}$

As reported in international studies ${ }^{[6]}$ UTI rates in our study increased with subsequent readmissions (12\%, 25\% and $40 \%$ in the first, second and third readmissions, respectively). People with complete SCI and cervical injuries are prone to UTIs as a result of the use of indwelling catheters rather than clean intermittent self-catheterisation. ${ }^{[10]}$ Deteriorating neurological status includes deterioration of motor function or sensation, and it accounted for $6 \%$ of the readmissions in this study. Little is known about worsening neurological status in PWSCI and its potential to lead to readmission to hospital. LOS to treat deteriorating neurological status ranged from 5 to 167 days in hospital, with LOS varying according to the type of function loss. ${ }^{[2]}$ Although respiratory complications were only the fifth most common reason for readmission, they are 
potentially life threatening. In our study, $4 \%$ of total readmissions were due to respiratory complications. There were individual cases of acute pulmonary insufficiency, bronchopneumonia, chest pains and pulmonary tuberculosis. This is very different from the recent local study by Joseph and Nilsson Wikmar, ${ }^{[8]}$ who attributed respiratory causes to $24 \%$ of SHCs. In our study, the respiratory complications were evenly distributed between paraplegia and tetraplegia $(50 \%$ each), with no respiratory complications in lower paraplegics (T7 S5). We found no evidence for increased susceptibility to respiratory complications in patients with tetraplegia, and there was an equal occurrence of respiratory complications in patients with $\mathrm{C} 1-\mathrm{C} 4$, C5 - C8 and T1 - T6 NLI.

The probability of initial readmission and subsequent admissions was found to be associated with type of SCI, completeness of SCI and NLI, similar to a Taiwanese study ${ }^{[11]}$ These findings suggest that the injury profile remains an important factor in readmission of PWSCI to the hospital setting. Our study investigated LOS once patients were readmitted and did not investigate LOS during the actual rehabilitation process. More research is needed to determine whether PWSCI were ready for initial hospital discharge, with the purpose of preventing subsequent readmissions.

The weakness of the present study is that only one database (from a private rehabilitation facility) was investigated. It is possible that a database from a state rehabilitation facility would have provided different results. However, the study has helped to address the literature gap regarding readmission in SA. The findings can be used in clinical practice to develop prevention programmes to decrease the occurrence of SHCs and ultimately reduce the chance of readmission. This study found that there are non-modifiable risk factors for readmission. Focused SCI education is needed to empower PWSCI by informing them about SHCs specific to their type, completeness and level of injury.

\section{Conclusions}

In this study, PWSCI were readmitted to hospital after rehabilitation as a result of SHCs including skin, urinary, nervous, digestive and respiratory complications. These complications are ubiquitous in PWSCIs, and in our study the most common SHCs were PUs, UTIs and deteriorating neurological status. Patients with paraplegia, particularly at NLI T1 - T6, and those with an incomplete injury had greater odds of readmission than their counterparts. The identified risk factors are non-modifiable, and warrant specific health education and preventive programmes for these patients. The high readmission rates due to SHCs in a private, specialised SCI rehabilitation facility in this study underscore that we are still far from achieving success in preventing these conditions. Readmission rates need to be reduced, and further research in the SA context is required to set up protocols and models to achieve this.

Declaration. None.

Acknowledgements. We thank Summit Rehab Meulmed for granting permission to use their database. We also thank Dr Cheryl A Tosh for editing assistance.

Author contributions. MKM was the primary researcher, conceptualised the study, collected data, interpreted results and wrote the paper. SASO was the biostatistician who converted the Excel data into Stata format, analysed the data and assisted in interpretation of the results. As the researcher's supervisor, JM was involved in conceptualising the study and critically reviewed the manuscript.

Funding. None.

Conflicts of interest. MKM was an employee of Summit Rehab Meulmed from August 2012 to August 2014, of which 4 months constituted the period researched in this study. There are no further relationships that might have influenced the authors in writing this article.

\footnotetext{
1. Young A, Webster B, Giunti G, et al. Rehospitalization following compensable work-related tetraplegia. Spinal Cord 2006;44(6):374-378. https://doi.org/10.1038/sj.sc.3101858

2. De Jong G, Tian W, Hsieh C-H, et al. Rehospitalization in the first year of traumatic spinal cord injury after discharge from medical rehabilitation. Arch Phys Med Rehabil 2013;94(4 Suppl 2):587-597. https://doi.org/10.1016/j.apmr.2012.10.037

3. Kalpakjian CZ, Scelza WM, Forchheimer MB, et al. Preliminary reliability and validity of a spinal cord injury secondary conditions scale. J Spinal Cord Med 2007;30(2):131-139. https://www.ncbi.nlm.nih. gov/pmc/articles/PMC2031942/ (accessed 7 January 2019).

4. Krause JS, Saunders LL. Risk of hospitalizations after spinal cord injury: Relationship with biographical, injury, educational, and behavioral factors. Spinal Cord 2009;47(9):692-697. https://doi org/10.1038/sc.2009.16

5. Jensen MP, Molton IR, Groah SL, et al. Secondary health conditions in individuals aging with SCI Terminology, concepts and analytic approaches. Spinal Cord 2012;50(5):373-378. https://doi. org/10.1038/sc.2011.150

6. Cardenas DD, Hoffman JM, Kirshblum S, et al. Etiology and incidence of rehospitalization after traumatic spinal cord injury: A multicenter analysis. Arch Phys Med Rehabil 2004;85(11):1757-1763. traumatic spinal cord injury: A multicenter
https://doi.org/10.1016/j.apmr.2004.03.016

7. Mathew A, Samuelkamaleshkumar S, Radhuka S, et al. Engagement in occupational activities and Mathew A, Samuelkamaleshkumar S, Radhuka S, et al. Engagement in occupational activities and
pressure ulcer development in rehabilitated South Indian persons with spinal cord injury. Spinal Cord 2013;51(2):150-155. https://doi.org/10.1038/sc.2012.112

8. Joseph C, Nilsson Wikmar L. Prevalence of secondary medical complications and risk factors for pressure ulcers after traumatic spinal cord injury during acute care in South Africa. Spinal Cord 2016;54(7):535-539. https://doi.org/10.1038/sc.2015.189

9. Adriaansen JJE, Ruijs LEM, van Koppenhagen CF, et al. Secondary health conditions and quality of life in persons living with spinal cord injury for at least ten years. J Rehabil Med 2016;48(10):853-860. https://doi.org/10.2340/16501977-2166

10. Liu C-W, Attar KH, Gall A, et al. The relationship between bladder management and health-related quality of life in patients with spinal cord injury in the UK. Spinal Cord 2010;48(4):319-324. https:// doi.org/10.1038/sc.2009.132

11. Lam C, Chen P-L, Kang J-H, et al. Risk factors for 14-day rehospitalisation following trauma with new traumatic spinal cord injury diagnosis: A 10-year nationwide study in Taiwan. PLoS One 2017;12(9):e0184253. https://doi.org/10.1371/journal.pone.0184253
}

Accepted 16 June 2018 